\title{
Front Matter: Volume 7935
}

, "Front Matter: Volume 7935," Proc. SPIE 7935, Organic Photonic Materials and Devices XIII, 793501 (20 April 2011); doi: 10.1117/12.894804

SPIE. Event: SPIE OPTO, 2011, San Francisco, California, United States 


\section{PROCEEDINGS OF SPIE}

\section{Organic Photonic Materials \\ and Devices XIII}

Robert L. Nelson

François Kajzar

Toshikuni Kaino

Yasuhiro Koike

Editors

24-26 January 2011

San Francisco, California, United States

Sponsored and Published by

SPIE

Volume 7935 
The papers included in this volume were part of the technical conference cited on the cover and title page. Papers were selected and subject to review by the editors and conference program committee. Some conference presentations may not be available for publication. The papers published in these proceedings reflect the work and thoughts of the authors and are published herein as submitted. The publisher is not responsible for the validity of the information or for any outcomes resulting from reliance thereon.

Please use the following format to cite material from this book:

Author(s), "Title of Paper," in Organic Photonic Materials and Devices XIII, edited by Robert L. Nelson, François Kajzar, Toshikuni Kaino, Yasuhiro Koike, Proceedings of SPIE Vol. 7935 (SPIE, Bellingham, WA, 2011) Article CID Number.

ISSN 0277-786X

ISBN 9780819484727

Published by

SPIE

P.O. Box 10, Bellingham, Washington 98227-0010 USA

Telephone +1 3606763290 (Pacific Time) · Fax +1 3606471445

SPIE.org

Copyright (c) 2011, Society of Photo-Optical Instrumentation Engineers

Copying of material in this book for internal or personal use, or for the internal or personal use of specific clients, beyond the fair use provisions granted by the U.S. Copyright Law is authorized by SPIE subject to payment of copying fees. The Transactional Reporting Service base fee for this volume is $\$ 18.00$ per article (or portion thereof), which should be paid directly to the Copyright Clearance Center (CCC), 222 Rosewood Drive, Danvers, MA 01923. Payment may also be made electronically through CCC Online at copyright.com. Other copying for republication, resale, advertising or promotion, or any form of systematic or multiple reproduction of any material in this book is prohibited except with permission in writing from the publisher. The CCC fee code is 0277-786X/11/\$18.00.

Printed in the United States of America.

Publication of record for individual papers is online in the SPIE Digital Library.

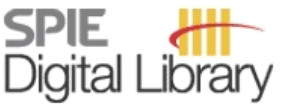

SPIEDigitalLibrary.org

Paper Numbering: Proceedings of SPIE follow an e-First publication model, with papers published first online and then in print and on CD-ROM. Papers are published as they are submitted and meet publication criteria. A unique, consistent, permanent citation identifier (CID) number is assigned to each article at the time of the first publication. Utilization of CIDs allows articles to be fully citable as soon as they are published online, and connects the same identifier to all online, print, and electronic versions of the publication. SPIE uses a six-digit CID article numbering system in which:

- The first four digits correspond to the SPIE volume number.

- The last two digits indicate publication order within the volume using a Base 36 numbering system employing both numerals and letters. These two-number sets start with 00, 01, 02, 03, 04 , 05, 06, 07, 08, 09, OA, OB ... 0Z, followed by 10-1Z, 20-2Z, etc.

The CID number appears on each page of the manuscript. The complete citation is used on the first page, and an abbreviated version on subsequent pages. Numbers in the index correspond to the last two digits of the six-digit CID number. 


\section{Contents}

vii Conference Committee

\section{SESSION 1 NONLINEAR OPTICS}

793502 Optimization of organic NLO materials for integration with silicon photonic, plasmonic (metal optics), and metamaterial devices (Keynote Paper) [7935-01]

L. R. Dalton, A. K.-Y. Jen, B. H. Robinson, R. Overney, S. J. Benight, D. B. Knorr, Jr., Univ. of Washington (United States); S. Kim, H. R. Fetterman, Univ. of California Los Angeles

(United States); C. Zhang, Norfolk State Univ. (United States)

\section{SESSION 2 ORGANIC LED}

793509 Hybrid organic light emitting device with silicon-rich oxide as hole transporting layer [7935-08]

G. Z. Ran, D. F. Jiang, Y. Yin, W. J. Xu, Peking Univ. (China)

\section{SESSION 3 ORGANIC LASER AND EMISSION}

7935 OA Towards highly-efficient liquid crystal microlasers (Invited Paper) [7935-09]

F. Araoka, H. Takezoe, Tokyo Institute of Technology (Japan)

7935 OB Characterization of fluorescence resonance energy transfer of a nanopatterned fluorophore doped polymer film fabricated using nanoimprint lithography [7935-10] R. Furukawa, The Univ. of Electro-Communications (Japan)

7935 OC Energy transfer processes in a polymer laser [7935-11]

K. H. Ibnaouf, Imam Mohammed bin Saud Islamic d Univ. (Saudi Arabia); M. S. Alsalhi, V. Masilamani, Z. S. Abu Mustafa, King Saud Univ. (Saudi Arabia)

7935 OD Laser from a liquid excimer [7935-12] M. Al Salhi, King Saud Univ. (Saudi Arabia); K. H. Ibanouf, Imam Mohammed bin Saud Islamic d Univ. (Saudi Arabia); V. Masilamani, King Saud Univ. (Saudi Arabia)

7935 OE Preparation and characterization of DNA-aromatic surfactant complexes for optoelectronic applications [7935-13]

T.-Y. Lin, C.-Y. Chang, C.-H. Lien, Y.-W. Chiu, W.-T. Hsu, C.-H. Su, Y.-S. Wang, Y.-C. Hung, National Tsing Hua Univ. (Taiwan) 
7935 OG Effect of intermolecular interactions on charge and exciplex formation in high-performance organic semiconductors [7935-15]

W. E. B. Shepherd, A. D. Platt, G. Banton, D. Hofer, Oregon State Univ. (United States); M. A. Loth, J. E. Anthony, Univ. of Kentucky (United States); O. Ostroverkhova, Oregon State Univ. (United States)

$7935 \mathrm{OH}$ Charge transport anisotropy in a pentacene transistor with an underlying photo-alignment layer [7935-16]

T. Kawaguchi, T. Okura, Y. Kondo, I. Fujieda, Ritsumeikan Univ. (Japan)

\section{SESSION 5 PHOTONIC TECHNOLOGIES}

7935 0J Photonic polymer research based on strategic promotion of innovative research and development supported by Japan Science and Technology Agency (Keynote Paper) [7935-18]

S. Miyata, Tokyo Institute of Technology (Japan) and Japan Science and Technology Agency (Japan); N. Tsutsumi, Kyoto Institute of Technology (Japan); J. Watanabe, Tokyo Institute of Technology (Japan); T. Yatagai, Utsunomiya Univ. (Japan); O. Sugihara, Tohoku Univ. (Japan); K. Hakuta, The Univ. of Electro-Communications (Japan)

$7935 \mathrm{OL}$ Template assisted polymerization of functional materials and their opto-electronic properties [7935-20]

J. Kim, Yonsei Univ. (Korea, Republic of); C. Anand, National Institute for Materials Science (Japan); J. You, Y. Kim, B. Kim, Yonsei Univ. (Korea, Republic of); A. Vinu, National Institute for Materials Science (Japan); E. Kim, Yonsei Univ. (Korea, Republic of)

7935 OM Fabrication and characterization of PDMS thin film [7935-21]

S. Wang, A. Kallur, Univ. of North Texas (United States); A. Goshu, Total Wire Corp.

(United States)

\section{SESSION 6 NANO PHOTONICS}

$7935 \mathrm{OP}$ Effects of surface chemistry and shape on nonlinear absorption, scattering, and refraction of PbSe nanocrystals [7935-24]

I. L. Bolotin, D. J. Asunskis, A. M. Jawaid, Y. Liu, P. T. Snee, L. Hanley, Univ. of Illinois at Chicago (United States) 
7935 OS Multifunctional hybrid nanoparticles for two-photon fluorescence imaging and photodynamic therapy (Invited Paper) [7935-28]

P. L. Baldeck, Lab. Interdisciplinaire de Physique, CNRS, Univ. Joseph Fourier (France) and Lab. de Chimie, CNRS, Univ. de Lyon 1 (France); M. Maurin, Lab. Interdisciplinaire de Physique, CNRS, Univ. Joseph Fourier (France); C. Philipot, Institut Néel, CNRS, Univ. Joseph Fourier (France); S. Zaiba, Lab. Interdisciplinaire de Physique, CNRS, Univ. Joseph Fourier (France) and Univ. M'hamed Bouguera (Algeria); T. Gallavardin, O. Maury, C. Andraud, Lab. de Chimie, CNRS, Univ. de Lyon 1 (France); F. Dubois, A. Ibanez, Institut Néel, CNRS, Univ. Joseph Fourier (France); F. Lerouge, S. Parola, Lab. de Chimie, CNRS, Univ. de Lyon 1 (France); O. Stephan, Lab. Interdisciplinaire de Physique, CNRS, Univ. Joseph Fourier (France); B. van Der Sanden, INSERM (France)

\section{SESSION 8 NEW MATERIALS AND METHODS}

7935 OT New developments in molecular photonics: from photoswitchable nonlinearities to nonlinear nanoplasmonics (Keynote Paper) [7935-30]

I. Ledoux-Rak, K. Hoang-Thi, Anu, J. Zyss, Ecole Normale Supérieure de Cachan (France)

7935 OU Ferromagnetism in pristine polythiophene at low temperature (Invited Paper) [7935-31] P. Gangopadhyay, The Univ. of Arizona (United States); G. Koeckelberghs, Katholieke Univ. Leuven (Belgium); A. Persoons, The Univ. of Arizona (United States)

$7935 \mathrm{OV}$ Chirality in molecular films at the air-water interface [7935-32]

E. Benichou, G. Gassin-Martin, A. Derovet, I. Russier-Antoine, G. Bachelier, C. Jonin, N. Lascoux, Lab. de Spectrométrielonique et Moléculaire, CNRS, Univ. Claude Bernard Lyon 1 (France); M. Liu, Beijing National Lab. for Molecular Sciences (China); P.-F. Brevet, Lab. de Spectrométrielonique et Moléculaire, CNRS, Univ. Claude Bernard Lyon 1 (France)

\section{SESSION 9 APPLICATIONS}

7935 OY Switching mechanism of an optically-gated optical switch using an organic dye [7935-35] T. Hiraga, I. Ueno, National Institute of Advanced Industrial Science and Technology (Japan); N. Shiga, Trimatiz Ltd. (Japan); H. Watanabe, S. Futaki, Inter Energy Co., Ltd. (Japan); M. Kubo, Himeji Rika Co., Ltd. (Japan); S. Takarada, N. Tanaka, Dainichiseika Color \& Chemicals Mfg. Co., Ltd. (Japan)

$79350 Z$ Enhanced laser induced polarization switching and holography using a dynamic holographic network recording system [7935-36] Q. Sun, P. WU, New Span Opto-Technology Inc. (United States); S. Baig, H. LU, M. R. Wang, Univ. of Miami (United States)

793510 Optical data storage in photochromic compounds [7935-37]

W. Dallari, Italian Institute of Technology (Italy) and Univ. of Genoa (Italy); M. Scotto d'Abbusco, E. Samoylova, F. Pignatelli, A. Athanassiou, R. Cingolani, Italian Institute of Technology (Italy); A. Diaspro, Italian Institute of Technology (Italy) and Univ. of Genoa (Italy); M. Allione, Italian Institute of Technology (Italy) 
793515 Improvement in viewing angle properties of top-emitting organic light-emitting devices [7935-42]

B.-T. Liou, Hsiuping Institute of Technology (Taiwan); M.-C. Tsai, Y.-H. Huang, F.-M. Chen, Y.-R. Lin, Y.-K. Kuo, National Changhua Univ. of Education (Taiwan)

793516 Photopatterning and electro-optical switching of redox active fluorescent polymers [7935-43]

S. Seo, Y. Kim, J. You, T. Park, E. Kim, Yonsei Univ. (Korea, Republic of)

793517 Ultrafast nonlinear optical properties and excited state dynamics of phthalocyanine thin films [7935-44]

S. Venugopal Rao, P.T. Anusha, T. S. Prashant, D. Swain, S. P. Tewari, Univ. of Hyderabad (India)

793518 Synthesis and characterization of thiophene-based conjugated copolymer containing ferroceneunits for organic electronics [7935-45]

S. Kim, Y. Kim, X. Yang, K. Kim, W. Kim, E. Kim, Yonsei Univ. (Korea, Republic of)

Author Index 


\title{
Conference Committee
}

\author{
Symposium Chair
}

Liang-Chy Chien, Kent State University (United States)

Symposium Cochairs

Klaus P. Streubel, OSRAM GmbH (Germany)

E. Fred Schubert, Rensselaer Polytechnic Institute (United States)

Program Track Chair

James G. Grote, Air Force Research Laboratories (United States)

Conference Chairs

Robert L. Nelson, Air Force Research Laboratory (United States)

François Kajzar, Université d'Angers (France)

Toshikuni Kaino, Tohoku University (Japan)

Yasuhiro Koike, Keio University (Japan)

Program Committee

Chantal Andraud, Ecole Normale Supérieure de Lyon (France)

Werner J. Blau, Trinity College Dublin (Ireland)

Christoph Bubeck, Max-Planck-Institut für Polymerforschung (Germany)

Fabrice Charra, Commissariat à l'Énergie Atomique (France)

Darnell E. Diggs, Air Force Research Laboratory (United States)

Alain F. Fort, Institut de Physique et Chimie des Matériaux de Strasbourg (France)

James G. Grote, Air Force Research Laboratory (United States)

F. Kenneth Hopkins, Air Force Research Laboratory (United States)

Alex K. Y. Jen, University of Washington (United States)

Michael H. C. Jin, The University of Texas at Arlington (United States)

Eunkyoung Kim, Yonsei University (Korea, Republic of)

Jang-Joo Kim, Seoul National University (Korea, Republic of)

Nakjoong Kim, Hanyang University (Korea, Republic of)

Junya Kobayashi, NTT Photonics Laboratory (Japan)

Isabelle N. Ledoux-Rak, École Normale Supérieure de Cachan

(France)

Charles Y. C. Lee, Air Force Office of Scientific Research (United States)

Kwang-Sup Lee, Hannam University (Korea, Republic of)

Emisoon Mah, Air Force Research Laboratory (United States) 
Seth R. Marder, Georgia Institute of Technology (United States)

Antoni Cz. Mitus, Wroclaw University of Technology (Poland)

Robert A. Norwood, College of Optical Sciences, The University of Arizona (United States)

Jean-Michel Nunzi, Queen's University (Canada)

Susanna Orlic, Technische Universität Berlin (Germany)

Ileana Rau, Polytechnical University of Bucharest (Romania)

Niyazi Serdar Sariciftci, Johannes Kepler University of Linz (Austria)

Kenneth D. Singer, Case Western Reserve University (United States)

Don J. Smith, U.S. Air Force (United Kingdom)

Rebecca E. Taylor, Lockheed Martin Space Systems Company (United States)

Toshiyuki Watanabe, Tokyo University of Agriculture and Technology (Japan)

Shiyoshi Yokoyama, Kyushu University (Japan)

Session Chairs

$1 \quad$ Nonlinear Optics

Robert L. Nelson, Air Force Research Laboratory (United States)

2 Organic LED

Robert L. Nelson, Air Force Research Laboratory (United States)

3 Organic Laser and Emission

Chantal Andraud, Ecole Normale Supérieure de Lyon (France)

$4 \quad$ Organic Electronics

Jang-Joo Kim, Seoul National University (Korea, Republic of)

$5 \quad$ Photonic Technologies

Yasuhiro Koike, Keio University (Japan)

6 Nano Photonics

Toshikuni Kaino, Tohoku University (Japan)

$7 \quad$ Nonlinear Absorption

Kwang-Sup Lee, Hannam University (Korea, Republic of)

8 New Materials and Methods

François Kajzar, Université d'Angers (France)

9 Applications

André P. Persoons, Katholieke Universiteit Leuven (Belgium) 